\title{
ALMOST COMPLEX STRUCTURES ON COMPLEX PROJECTIVE SPACES
}

BY

\section{ALAN THOMAS}

ABSTRACT. In this paper we classify the almost complex structures on a complex projective space as roots of a certain polynomial equation.

Introduction. A real oriented vector bundle $E$ is said to admit an almost complex structure if there is a complex vector bundle $F$ such that $E \cong F$ as oriented vector bundles. An almost complex structure on $E$ is a (complex) isomorphism class of such bundles $F$. When $X$ is a $2 n$-dimensional oriented manifold and $T X$ is its tangent bundle, an almost complex structure on $T X$ is called an almost complex structure on $X$, and $X$ together with an almost complex structure is called an almost complex manifold. A special case of this is when $X$ is a complex manifold with complex tangent bundle $\mathcal{T} X$. Since $\left(\mathcal{T}_{X}\right)_{R}=T X, X$ always admits an almost complex structure.

In this paper we study the generic complex manifolds, the complex projective spaces, and classify their almost complex structures as roots of a certain polynomial equation. In this context we give an answer to a question of Hirzebruch [7] posed in 1954 concerning which elements of cohomology arise as Chern classes of almost complex structures. The techniques used to solve this problem apply equally well in other cases (for example to $U(n), s^{2 n}, G_{2}\left(C^{n}\right)=$ Grassmannian of 2-planes in $C^{n}, s^{2 m+1} \times L^{n}(p)$-where $L^{n}(p)$ is the lens space and $p$ is sufficiently large, various products of these, etc.) and the details and calculations in these cases will appear later. For example, one can easily show that $s^{2 n+1} \times$ $s^{2 m+1}$ admits precisely one almost complex structure. (In fact this is the complex tangent bundle of the complex structure defined by Calabi and Eckmann [9].)

1. Definitions and statement of main theorems. Let $\mathbf{Z}^{m}$ be the product of $m$ copies of the integers as an abelian group. We consider $\mathbf{Z}^{m} \subset \mathbf{Z}^{m+1}$ by the inclusions $\left(a_{1}, \ldots, a_{m}\right) \mapsto\left(a_{1}, \cdots, a_{m}, 0\right)$, and put $Z^{\infty}=\bigcup_{m \geq 1} Z^{m}$. For each $m$, let $p_{m}: \mathrm{Z}^{\infty} \rightarrow \mathrm{Z}$ be projection onto the $m$ th coordinate.

Received by the editors December 13, 1972 and, in revised form, July 9, 1973. AMS (MOS) subject classifications (1970). Primary 53C15; Secondary 32C10, 55B15. Key words and phrases. Almost complex, vector bundle, complex projective space. 
Let $t \in H^{2}\left(\mathbf{C P}^{m}\right)$ be the cohomology class corresponding to the submanifold $\mathbf{C P}^{m-1}$ via the natural orientation of $\mathrm{CP}^{m}$. The abelian group $\tilde{H}^{e v}\left(\mathrm{CP}^{m}\right)$ is free with generators $t, t^{2}, \ldots, t^{m}$. For convenience of notation we identify this group with $\mathrm{Z}^{m}$ by means of the isomorphism $a_{1} t+a_{2} t^{2}+\cdots+a_{m} t^{m} \mapsto\left(a_{1}, a_{2}, \ldots, a_{m}\right)$.

Definition. The $k$ th Newton function $s_{k}: Z^{\infty} \rightarrow Z$ is defined inductively by

(i) $s_{1}=p_{1}$,

(ii) $s_{k}(a)=p_{1}(a) s_{k-1}(a)-p_{2}(a) s_{k-2}(a)+\cdots+(-1)^{k-1} p_{k-1}(a) s_{1}(a)+$ $(-1)^{k} k p_{k}(a)$.

We notice that $s_{k}(a)$ is a polynomial in $p_{1}(a), \cdots, p_{k}(a)$, the first $k$ coordinates of $a$. Inductively we define a homomorphism $f_{k}: \mathrm{Z}^{k} \rightarrow \mathrm{Z}$ by

(i) $f_{1}=$ identity,

(ii) $f_{k}\left(a_{1}, \ldots, a_{k}\right)=f_{k-1}\left(a_{2}, \ldots, a_{k}\right)-(k-1) f_{k-1}\left(a_{1}, \ldots, a_{k-1}\right)$.

Theorem A. The n-ple $a=\left(a_{1}, \ldots, a_{n}\right)$ is the Chern vector of a vector bundle over $\mathrm{CP}^{n}$ iff $f_{k}\left(s_{1}, \ldots, s_{k}\right)=0 \bmod k !$ for $1 \leq k \leq n$ where $s_{i}=s_{i}(a)$.

For $n$ a positive integer let

$$
\begin{aligned}
r(n)=r & =\text { largest integer } \leq 1 / 2 n, \\
r^{\prime}(n)=r^{\prime} & =\text { smallest integer } \geq 1 / 2 n, \\
u(n)=u & =0 \text { if } n \text { is even, } \\
& =1 \text { if } n \equiv 3 \bmod 4, \\
& =2 \text { if } n \equiv 1 \bmod 4 .
\end{aligned}
$$

Define $f^{n}: \mathrm{Z}^{\prime} \rightarrow \mathrm{Z}[[t]]$ (the ring of formal power series over the integers) by the equation

$$
f^{n}\left(x_{1}, \cdots, x_{r^{\prime}}\right)=(1+t)^{n+1}\left(1+(n-1) ! t^{n}\right)^{u x_{r^{\prime}}} \prod_{1 \leq k \leq r}\left(\frac{1+k t}{1-k t}\right)^{x_{k}}
$$

and let $f_{m}^{n}\left(x_{1}, \ldots, x_{r^{\prime}}\right)$ be the coefficient of $t^{m}$, that is

$$
f^{n}\left(x_{1}, \cdots, x_{r^{\prime}}\right)=\sum_{m} f_{m}^{n}\left(x_{1}, \cdots, x_{r^{\prime}}\right) t^{m} .
$$

We note that $f_{m}^{n}\left(x_{1}, \cdots, x_{r}\right)$ is a polynomial of degree less than or equal to $m$.

Theorem B. There is a 1-1 correspondence between the set of almost complex structures on $\mathrm{CP}^{n}$ and the set of solutions of the equation $f_{n}^{n}\left(x_{1}, \cdots, x_{r}\right)=$ $n+1$. Moreover, the almost complex structure corresponding to the solution $\left(a_{1}, \ldots, a_{r^{\prime}}\right)$ has ith Chern class $f_{i}^{n}\left(a_{1}, \ldots, a_{r^{\prime}}\right)$ so that the 'total Chern class' is precisely $f^{n}\left(a_{1}, \ldots, a_{r^{\prime}}\right)$ truncated after the nth term.

Inductively we define a homomorphism $g_{k}: \mathrm{Z}^{k} \rightarrow \mathrm{Z}$ by

(i) $g_{1}=$ identity, 
(ii) $g_{k}\left(a_{1}, \ldots, a_{k}\right)=g_{k-1}\left(a_{2}, \ldots, a_{k}\right)-(k-1)^{2} g_{k-1}\left(a_{1}, \ldots, a_{k-1}\right)$.

Theorem $\mathrm{C}$. The n-ple $a=\left(a_{1}, \cdots, a_{n}\right)$ is the Chern vector of an almost complex structure on $\mathrm{CP}^{n}$ iff

(1) $a_{1}=n+1 \bmod 2$;

(2) $a_{n}=n+1$

(3) $g_{k}\left(s_{1}, s_{3}, \cdots, s_{2 k-1}\right)=0 \bmod 2((2 k-1) !), 2 \leq k \leq r$;

(4) if $n$ is odd, $g_{r},\left(s_{1}, \cdots, s_{n}\right)=0 \bmod u(n !)$;

(5) $s_{2 k}=n+1$ for $1 \leq k \leq r$;

where $s_{i}=s_{i}(a)$.

2. Preliminaries. We recall briefly from [2] the functors $\tilde{G}$ and $\tilde{H}$ and their relation with $\tilde{K}$-theory. The reader is referred to this paper for explicit and further details.

For $X$ a finite connected based CW-complex, $\tilde{G}(X)$ is the abelian group whose elements are formal expressions

$$
1+a_{1}+\cdots+a_{i}+\cdots \text { where } a_{i} \in H^{2 i}(X)
$$

with addition $\oplus$ defined by

$$
\left(1+a_{1}+\cdots\right) \oplus\left(1+b_{1}+\cdots\right)=1+c_{1}+\cdots
$$

where $c_{i}=a_{i}+a_{i-1} b_{1}+a_{i-2} b_{2}+\cdots+b_{i}$.

If $E$ is a $C^{n}$-bundle over $X$ with $i$ th Chern class $c_{i}(E) \in H^{2 i}(X)$ then the 'total Chern class' of $E$

$$
\tilde{c}(E)=1+c_{1}(E)+\cdots+c_{n}(E)
$$

is an element of $G(X)$, and since if $F$ is a $\mathrm{C}^{m}$-bundle over $X \tilde{c}(E \oplus F)=\tilde{c}(E) \oplus$ $\tilde{c}(F)$, we see that $\tilde{c}$ induces a natural homomorphism

$$
\tilde{c}: \tilde{K}(X) \rightarrow \tilde{G}(X) \text {. }
$$

Then $\tilde{c}$ is an isomorphism modulo finite groups. (In fact a much stronger result is true [2].) The image of $\tilde{c}$ is the subgroup of 'vectors' which occur as total Chern class of a vector bundle.

Let $\tilde{H}(X)=\bigoplus_{n} \tilde{H}^{2 n}(X)$ as an abelian group. We give $\tilde{H}(X)$ the structure of a graded ring with product denoted by juxtaposition defined in terms of cup product by the formula

$$
x_{m} x_{n}=\frac{(m+n) !}{(m !)(n !)} x_{m} \cup x_{n} \text { where } x_{i} \in H^{2 i}(X) .
$$

There is a natural homomorphism $\sigma: \widetilde{G}(X) \rightarrow \widetilde{H}(X)$ which is defined in terms of Newton polynomials with the property that if $a \in H^{2}(X)$ then $\left.d 1+a\right)=\left(a, a^{2}, \ldots, a^{n}\right)$. 
We define $\tilde{s}: \tilde{K}(X) \rightarrow \tilde{H}(X)$ to be the composite $\tilde{s}=\tilde{\sigma c}$, which is a ring homomorphism. If $E$ is a complex vector bundle over $X$, then the $i$ th term $s_{i}$ of $\tilde{s}(E-\operatorname{dim} E)$ is related to the Chern classes $c_{i}$ by the formula

$$
s_{i}-c_{1} s_{i-1}+\cdots+(-1)^{k} k c_{k}=0 \text {. }
$$

Both $\sigma$ and $\tilde{s}$ are isomorphisms modulo finite groups.

3. Proof of Theorem A. We now consider the case $X=\mathrm{CP}^{n}$. Let $H$ be the line bundle over $\mathrm{CP}^{n}$ defined by

$$
H=\left\{(V, v) \in \mathbf{C P}^{n} \times \mathbf{C}^{n+1}: v \in V\right\}
$$

where we think of $\mathrm{CP}^{n}$ as the set of 1-dimensional subspaces of $\mathrm{C}^{n+1}$. Let $\eta$ $=H-1 \in \widetilde{K}\left(\mathbf{C P}^{n}\right)$ and let $\eta_{R}$ be the image of $\eta$ under the 'realification' map

$$
R: \widetilde{K}(X) \rightarrow \widetilde{K O}(X) \text {. }
$$

The following theorem is well known. (See, for example, [3].)

3.1 Theorem. (1) $\widetilde{K}\left(\mathrm{CP}^{n}\right)$ is generated by $\eta, \eta^{2}, \ldots, \eta^{n}$ subject to the single relation $\eta^{n+1}=0$.

(2) $\widetilde{K O}\left(\mathrm{CP}^{n}\right)$ is generated by powers of $\eta_{R}$ subject to the relations

(i) if $n$ is even, $\left(\eta_{R}\right)^{r+1}=0$;

(ii) if $n \equiv 1 \bmod 4,2\left(\eta_{R}\right)^{r+1}=0$ and $\left(\eta_{R}\right)^{r^{\prime}+1}=0$;

(iii) if $n \equiv 3 \bmod 4,\left(\eta_{R}\right)^{r+1}=0$.

In particular, $\tilde{K}\left(\mathbf{C P}^{n}\right)$ is free abelian, and so is $\widetilde{K O}\left(\mathbf{C P}^{n}\right)$ provided $n \not 1$ $\bmod 4$. If $n \equiv 1 \bmod 4$, then $\widetilde{K O}\left(C^{n}\right)$ is the direct sum of a free abelian group and a copy of $Z_{2}$, so has a unique element of order 2 , namely $\left(\eta_{R}\right)^{r+1}$.

3.2 Lemma. $\tilde{G}\left(\mathbf{C P}^{n}\right)$ is a free abelian group of rank $n$.

Proof. Since $\tilde{G}\left(\mathbf{C P}^{n}\right)$ is isomorphic to $\tilde{K}\left(\mathbf{C P}^{n}\right)$ modulo finite groups, it is finitely generated, so it suffices to show it is torsion free. Suppose $a \in \tilde{G}\left(\mathbf{C P}^{n}\right)$ and $k \in \mathbf{Z}$ such that $k a=0$. Writing $a=1+a_{r}+$ higher terms where $a_{r} \in H^{2 r}\left(\mathbf{C P}^{n}\right)$ is nonzero, we see that

$$
k a=1+k a_{r}+\text { higher terms }
$$

so that $k=0$ since $H^{2 r}\left(\mathrm{CP}^{n}\right)$ is torsion free.

It follows that the maps

$$
\tilde{c}: \tilde{K}\left(\mathbf{C P}^{n}\right) \rightarrow \tilde{G}\left(\mathbf{C P}^{n}\right), \quad \sigma: \tilde{G}\left(\mathbf{C P}^{n}\right) \rightarrow \widetilde{H}\left(\mathbf{C P}^{n}\right), \quad \tilde{s}: \widetilde{K}\left(\mathbf{C P}^{n}\right) \rightarrow \widetilde{H}\left(\mathbf{C P}^{n}\right)
$$

are all monomorphisms. We identify $\tilde{G}\left(\mathrm{CP}^{n}\right)$ with $\mathrm{Z}^{n}$ by means of the bijection

$$
1+a_{1} t+\cdots+a_{n} t^{n} \mapsto\left(a_{1}, \cdots, a_{n}\right) .
$$


Let $C=\operatorname{Im} \tilde{c}$, and $S=\operatorname{Im} \tilde{s}$. Then $C$ can be identified with the set of elements $\left\{a \in \mathbf{Z}^{n}\right.$ such that $\left.\left(s_{1}(a), s_{2}(a), \ldots, s_{n}(a)\right) \in S\right\}$.

For $k \in \mathbf{Z}$, let $v_{k}=H^{k}-1 \in \tilde{K}\left(\mathbf{C P}^{n}\right)$. Then $\tilde{c}\left(v_{k}\right)=1+k t \in \tilde{G}\left(\mathbf{C P}^{n}\right)$ and $\tilde{s}\left(v_{k}\right)=\left(k, k^{2}, \ldots, k^{n}\right)$. Since $v_{k} \cdot v_{l}=v_{k+l}-v_{k}-v_{l}$, and $\eta=v_{1}$, one can show inductively that

$$
\eta^{k}=v_{k}-\left(\begin{array}{l}
k \\
1
\end{array}\right) v_{k-1}+\left(\begin{array}{l}
k \\
2
\end{array}\right) v_{k-2}-\cdots+(-1)^{k-1}\left(\begin{array}{c}
k \\
k-1
\end{array}\right) v_{1}
$$

and hence $\tilde{K}\left(\mathbf{Q P}^{n}\right)$ is generated by $v_{1}, \ldots, v_{n}$. So $S$ consists of all elements of the form

$$
a_{1} s\left(v_{1}\right)+a_{2} s\left(v_{2}\right)+\cdots+a_{n} s\left(v_{n}\right) \text { where } a_{i} \in \mathbf{Z}
$$

i.e. those elements $\left(b_{1}, \ldots, b_{n}\right) \in \mathrm{Z}^{n}$ such that the system of simultaneous equations

$$
\begin{gathered}
a_{1}+2 a_{2}+3 a_{3}+\cdots+n a_{n}=b_{1}, \\
a_{1}+2^{2} a_{2}+3^{2} a_{3}+\cdots+n^{2} a_{n}=b_{2}, \\
\vdots \\
a_{1}+2^{n} a_{2}+3^{n} a_{3}+\cdots+n^{n} a_{n}=b_{n}
\end{gathered}
$$

has a solution with $a_{i}$ integers.

3.3 Lemma. (i) $f_{i}\left(j, j^{2}, j^{3}, \ldots, j^{i}\right)=j ! /(j-i)$ !.

(ii) The augmented matrix of the above system of equations is row equivalent over the integers to the matrix $M$ defined by

$$
\begin{aligned}
M_{i j} & =0 \quad \text { if } i>j, \\
& =f_{i}\left(j, j^{2}, \ldots, j^{i}\right) \quad \text { for } i \leq j \leq n, \\
& =f_{i}\left(b_{1}, \cdots, b_{i}\right) \text { for } j=n+1 .
\end{aligned}
$$

Proof. Part (i) is proved by induction on $i$, starting with $f_{1}(j)=j=j ! /(j-1)$ !. Then

$$
f_{i+1}\left(j, \cdots, j^{i+1}\right)=f_{i}\left(j^{2}, \cdots, j^{i+1}\right)-i f_{i}\left(j, \cdots, j^{i}\right)=(j-i) f_{i}\left(j, \cdots, j^{i}\right)
$$

using the fact that $f_{i}$ is a homomorphism, and the result follows.

Part (ii) is proved using ( $i$ ) and by applying to the augmented matrix those row operations which inductively define $f_{i}$.

Since $M_{i i}=i$ ! by the lemma, we see that

$$
S=\left\{\left(b_{1}, \cdots, b_{n}\right): f_{i}\left(b_{1}, \cdots, b_{i}\right)=0 \bmod i ! \text { for } 1 \leq i \leq n\right\}
$$

which completes the proof of Theorem $A$. 
For $X$ a CW-complex, let $\operatorname{Vect}_{n}(X)$ be the set of (isomorphism classes of) $\mathrm{C}^{n}$-bundles over $X$. If $\operatorname{dim} X=2 n$, then $\operatorname{Vect}_{n}(X)$ is an abelian group. If $E, F \in$ Vect $_{n}(X)$, then by stability properties there is a $C^{n}$-bundle, say $E \cdot F$ determined uniquely up to isomorphism such that $E \oplus F=(E \cdot F) \oplus \mathbf{n}$, where $\mathrm{n}$ denotes the trivial $C^{n}$-bundle. The function $(E, F) \mapsto E \cdot F$ defines the group structure, and the natural map

$$
\operatorname{Vect}_{n}(X) \rightarrow K(X), \quad E \mapsto E-n
$$

is an isomorphism.

If $E \in \operatorname{Vect}_{n}\left(C P^{n}\right)$, then $\tilde{c}(E) \in C$, and this defines a function $f: \operatorname{Vect}_{n}\left(\mathbf{C P}^{n}\right)$ $\rightarrow C$. Conversely, if $a \in C$ then there is a $C^{n}$-bundle $E$ with $f(E)=a$. If $E^{\prime}$ is a second such $\mathrm{C}^{n}$-bundle, then $\tilde{c}\left(E-E^{\prime}\right)=0$ so $E-E^{\prime}=0$ in $\widetilde{K}\left(C P^{n}\right)$ and by stability, $E \cong E^{\prime}$ and the isomorphism class of $E$ is well defined. This defines a function $g: C \rightarrow \operatorname{Vect}_{n}\left(C P^{n}\right)$ such that $f g=1$.

3.4 Lemma. The maps $f: \operatorname{Vect}_{n}\left(\mathrm{CP}^{n}\right) \rightarrow C$ and $g: C \rightarrow \operatorname{Vect}_{n}\left(\mathrm{CP}^{n}\right)$ are inverse isomorphisms (of abelian groups).

This lemma together with Theorem $A$ classifies $\mathbf{C}^{n}$-bundles over $\mathbf{C P}^{\boldsymbol{n}}$. If $E$ is a $\mathrm{C}^{r}$-bundle over $\mathrm{CP}^{n}$ we say that $E$ extends over $\mathbf{C P}^{n+s}$ if there is a $\mathrm{C}^{r}$ bundle $E^{\prime}$ over $\mathrm{CP}^{n+s}$ which restricts to $E$. It is an interesting and unsolved problem to determine necessary and sufficient conditions for a $\mathbf{C}^{r}$-bundle over $\mathbf{C P}^{n}$ to extend over $\mathrm{CP}^{n+s}$. The following is a solution in the simplest case $G=n$, $s=1)$.

3.5 Proposition. A $\mathrm{C}^{n}$-bundle over $\mathrm{CP}^{n}$ extends over $\mathrm{CP}^{n+1}$ iff

$$
f_{n+1}\left(s_{1}, \cdots, s_{n+1}\right)=0 \bmod (n+1) \text { ! }
$$

where $s_{i}=s_{i}(c)$, and $c=\left(c_{1}(E), c_{2}(E), \ldots, c_{n}(E), 0\right)$.

Proof. Necessity is an easy corollary of Theorem A. Let $F$ be the $C^{n+1}$ bundle over $\mathrm{CP}^{n+1}$ with $c_{i}(F)=c_{i}(E)$ for $1 \leq i \leq n$, and $c_{n+1}(F)=0$. By hypothesis and Lemma 3.4, there is such a bundle. Over $C P^{n}$ we can write $F \simeq G \oplus 1$ by stability, and then by Lemma $3.4 G \cong E$ by examining Chern classes. So $F$ has a nonzero section over $C^{n}$ and since the Euler class of $F$ is zero this section extends to a nonzero section over $C^{n+1}$. So $F \cong E^{\prime} \oplus 1$ for some $E^{\prime}$ an $n$ dimensional bundle over $\mathrm{CP}^{n+1}$, and $E^{\prime}$ restricted to $\mathrm{CP}^{n}$ is $E$.

4. Almost complex structures. If $X$ is a $2 n$-dimensional oriented manifold, let

$$
e: \tilde{K}(X)=\operatorname{Vect}_{n}(X) \rightarrow H^{2 n}(X)=\mathbf{Z}
$$


be the function which associates to an $n$-dimensional complex vector bundle its nth Chern class, that is, its Euler class.

4.1 Proposition. If $X$ is a $2 n$-dimensional oriented manifold, there is a 1-1 correspondence between the set of almost complex structures on $X$ and the set of elements $x \in \tilde{K}(X)$ satisfying

(1) $e(x)=e(X)=$ Euler number of $X$;

(2) $R(x)=T X-2 n$ in $\widetilde{K O}(X)$, where $R: \widetilde{K}(X) \rightarrow \widetilde{K O}(X)$ is the realification homomorphism, and $T X$ is the tangent bundle of $X$.

Proof. If $A$ is an almost complex structure then $R(A-n)=T X-2 n$ and $e(A-n)$ $=c_{n}(A)=$ Euler class of $T X=$ Euler class of $X$. Conversely if $x \in \tilde{K}(X)$, let $A \epsilon$ $\operatorname{Vect}_{n}(X)$ be the corresponding element. Then $R(A-n)=T X-2 n$, so $A_{R} \simeq \simeq_{\text {stably }}$ $T X$, whence $A_{R} \oplus 1 \cong T X \oplus 1$ so $A_{R} \cong T X$ since they have the same Euler class, which is the obstruction to removing the trivial line bundle.

4.2 Corollary. If $X$ is a $2 n$-dimensional oriented manifold such that

(1) $H^{2 j}(X)$ bas only torsion relatively prime to $(j-1)$ !,

(2) $\widetilde{K O}(X)$ bas no 2-torsion,

then there is a 1-1 correspondence between almost complex structures on $X$ and elements $E \in \operatorname{Vect}_{n}(x)$ such that

(a) $c_{2 i}(E \oplus \bar{E})^{n}=p_{i}(X)=i$ th Pontrjagin class, $1 \leq i \leq n$,

(b) $e(E)=e(X)$.

Proof. If $x \in \tilde{K}(X)$, then $R(X)=T X-2 n$ iff $C R(x)=C(T X)-2 n$ (where $C: \widetilde{K O}(X) \rightarrow \widetilde{K}(X)$ is the complexification homomorphism) since $\widetilde{K O}(X)$ has no 2-torsion and $R C$ is multiplication by 2. So $R(E-n)=T X-2 n$ iff $E \oplus \bar{E}-2 n$ $=C(T X)-2 n$, that is iff $E \oplus \bar{E}$ and $C(T X)$ are stably isomorphic. By a result of Peterson [4] under the conditions of the hypothesis, 2 vector bundles are stably isomorphic iff they have the same Chern classes, and the result follows.

A particular example of Corollary 4.2 is $X=C P^{n}$ for $n \neq 1 \bmod 4$.

4.3 Proposition. The kernel of $\left.R: \widetilde{K}\left(C^{n}\right) \rightarrow \widetilde{K O}\left(C^{n}\right)^{n}\right)$ is freely generated by

(1) $w_{1}, \cdots, w_{r}, n$ even;

(2) $w_{1}, \cdots, w_{r}, \eta^{n}, n \equiv 3 \bmod 4$

(3) $w_{1}, \cdots, w_{r}, 2 \eta^{n}, n \equiv 1 \bmod 4$ where $w_{k}=H^{k}-H^{-k}$.

Proof. This is an easy consequence of the theorems of Fujii on the structure of $\widetilde{K O}\left(C^{n}\right)([5],[6])$ and the fact that

$$
(\eta-\bar{\eta})(\eta+\bar{\eta})^{k}=w_{k+1}+\left(\text { linear combination of } w_{1}, \cdots, w_{k}\right)
$$

which is easily proved by induction starting with $k=0$. 
Let $\mathfrak{T}$ denote the complex tangent bundle of the complex manifold $\mathrm{CP}^{n}$ and let $T$ denote the real tangent bundle of the differentiable manifold $\mathrm{CP}^{n}$. Then $\mathcal{J}_{R} \cong T$, and so if $x \in \tilde{K}\left(C P^{n}\right), R(x)=T-2 n$ iff

$$
x=(\mathcal{T}-n)+a_{1} w_{1}+\cdots+a_{r} w_{r}+u a_{r^{\prime}} \eta^{n}
$$

for some integers $a_{1}, \ldots, a_{r}, a_{r}$. For such an $x$,

$$
\begin{aligned}
\tilde{c}(x) & =(1+t)^{n+1}\left(1+(n-1) ! t^{n}\right)^{u a_{r^{\prime}}} \prod_{1 \leq k \leq r}\left(\frac{1+k t}{1-k t}\right)^{a_{k}} \\
& =f^{n}\left(a_{1}, \cdots, a_{r^{\prime}}\right)
\end{aligned}
$$

and so $c(x)=n$th Chern class $=f_{n}^{n}\left(a_{1}, \ldots, a_{r^{\prime}}\right)$. So using Proposition 4.1 we see that any almost complex structure determines a solution of the equation

$$
f_{n}^{n}\left(x_{1}, \cdots, x_{r^{\prime}}\right)=n+1 \text {. }
$$

Conversely, if $\left(a_{1}, \ldots, a_{r}\right)$ is a solution, let

$$
x=(\mathcal{T}-n)+a_{1} w_{1}+\cdots+a_{r} w_{r}+u a_{r} \eta^{n}
$$

so that $R(x)=T$ and $e(x)=n+1$ so by Proposition $4.1 x$ determines an almost complex structure. This completes the proof of Theorem B.

The proof of Theorem $C$ now follows from Proposition 4.3 by methods similar to $\$ 3$. For an element $x \in \tilde{K}\left(C P^{n}\right)$ is of the form

$$
x=(\mathcal{J}-\mathbf{n})+a_{1} w_{1}+\cdots+a_{r} w_{r}+u a_{r} \eta^{n} \text { for some } a_{i}
$$

iff $s(x)=s(\mathcal{T}-n)+a_{1} s\left(w_{1}\right)+\cdots+a_{r} s\left(w_{i}\right)+u a_{r^{\prime}} s\left(\eta^{n}\right)$ for some $a_{i}$ iff the $n$ equations $(1 \leq i \leq n)$

$$
a_{1} s_{i}\left(w_{1}\right)+\cdots+a_{r} s_{i}\left(w_{r}\right)+a_{r} u s_{i}\left(\eta^{n}\right)=s_{i}(x)-s_{i}\left(I_{-n}\right)
$$

have a solution with $a_{i}$ integers. Since

$$
s\left(w_{k}\right)=2\left(k, 0, k^{3}, 0, k^{5}, 0, \ldots\right)
$$

we see that $s_{2 i}(x)=s_{2 i}(\mathcal{J}-\mathrm{n})=n+1$. The equations for $i$ odd are

$$
\begin{gathered}
2\left(a_{1}+2 a_{2}+\cdots+r a_{r}\right)=s_{1}(x)-(n+1), \\
2\left(a_{1}+2^{3} a_{2}+\cdots+r^{3} a_{r}\right)=s_{3}(x)-(n+1), \\
\vdots \\
2\left(a_{1}+2^{2 r-1} a_{2}+\cdots+r^{2 r-1} a_{r}\right)=s_{2 r-1}(x)-(n+1)
\end{gathered}
$$

and if $n$ is odd 


$$
2\left(a_{1}+2^{n} a_{2}+\cdots+r^{n} a_{r}\right)-u(n !) a_{r^{\prime}}=s_{n}(x)-(n+1) .
$$

Theorem $\mathrm{C}$ now follows from the following lemma.

4.4 Lemma. (i) $g_{i}\left(j, j^{3}, \ldots, j^{2 i-1}\right)=(j+i-1) ! /(j-i) !$.

(ii) The augmented matrix of the above system of equations is row equivalent over the integers to the matrix $N$ defined by

$$
\begin{aligned}
& N_{i j}=0 \text { if } i>j \text {, } \\
& =2 g_{i}\left(j, j^{3}, \ldots, j^{2 i-1}\right) \quad \text { for } i \leq j \leq r, \\
& \left.\begin{array}{l}
=g_{i}\left(s_{1}, s_{3}, \cdots, s_{2 i-1}\right) \text { for } 2 \leq i \text {, and } j=r^{\prime}+1 \\
=s_{1}-(n+1) \text { for } i=1, j=r^{\prime}+1
\end{array}\right\} \text { where } s_{i}=s_{i}(x),
\end{aligned}
$$

and if $n$ is odd

$$
\begin{aligned}
N_{i j} & =0 \quad \text { if } 1 \leq i \leq r, j=r^{\prime} \\
& =-2 u(n !), \quad i=j=r^{\prime}
\end{aligned}
$$

The proof of this lemma is similar to the proof of Lemma 3 with the additional observation that

$$
\begin{aligned}
g_{i}\left(s_{1}-(n+1), s_{3}\right. & \left.-(n+1), \ldots, s_{2 i-1}-(n+1)\right) \\
& =g_{i}\left(s_{1}, \ldots, s_{2 i-1}\right)-g_{i}(n+1, n+1, \ldots, n+1) \\
& =g_{i}\left(s_{1}, \ldots, s_{2 i-1}\right) \text { for } i \geq 2, \\
& =s_{1}-(n+1) \text { for } i=1 .
\end{aligned}
$$

\section{REFERENCES}

1. F. Hirzebruch, Neue topologische Methoden in der algebraischen Geometrie, Ergebnisse der Math. und ihrer Grenzgebiete, Heft 9, Springer-Verlag, Berlin, 1956; English transl., Die Grundlehren der math. Wissenschaften, Band 131, Springer-Verlag, New York, 1966. MR 18, 509; $34 \# 2573$.

2. A. Thomas, $A$ relation between $K$-theory and cohomology, Trans. Amer. Math. Soc. 193 (1974), 133-142.

3. B. J. Sanderson, Immersions and embeddings of projective spaces, Proc. London Math. Soc. (3) 14 (1964), 137-153. MR 29 \#2814.

4. F. P. Peterson, Some remarks on Chem classes, Ann. of Math. (2) 69 (1959), 414420. MR $21 \# 1593$. \#2005.

5. M. Fujii, $K_{U^{-g r o u p s}}$ of Dold manifolds, Osaka J. Math. 3 (1966), 49-64. MR 34 \#2143.

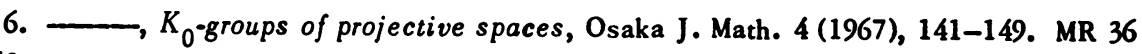

7. F. Hirzebruch, Some problems on differentiable and complex manifolds, Ann. of Math. (2) 60 (1954), 213-236. MR 16, 518. 
8. E. Thomas, Complex structures on real vector bundles, Amer. J. Math. 89 (1967), 887-908. MR $36 \# 3375$.

9. E. Calabi and B. Eckmann, $A$ class of compact complex manifolds which are not algebraic, Ann. of Math. (2) 58 (1953), 494-500.

10. M. F. Atiyah, K-theory, Lecture Notes by D. W. Anderson, 2nd. ed., Benjamin, New York, 1967. MR $36 \# 7130$.

11. D. Husemoller, Fibre bundles, McGraw-Hill, New York, 1966. MR 37 \#4821.

12. S. Hoggar, On KO-theory of Grassmannians, Quart. J. Math. Oxford Ser. (2) 20 (1969), 447-463. MR $40 \# 8048$.

13. R. Narasimhan, Analysis on real and complex manifolds, Advanced Studies in Pure Math., vol. 1, Masson, Paris; North-Holland, Amsterdam 1968. MR 40 \#4972.

14. T. Heaps, Almost complex structures on eight- and ten-dimensional manifolds, Topology 9 (1970), 111-119. MR 41 \#9283.

DEPARTMENT OF MATHEMATICS, UNIVERSITY COLLEGE OF SWANSEA, SINGLETON PARK, SWANSEA, WALES 